\title{
EFEITO DA ADUBAÇÃO ORGÂNICA NA PRODUÇÃO DE BIOMASSA EM QUEBRA-PEDRA (Phyllanthus stipulatus, EUPHORBIACEAE) EM MANAUS, AMAZONAS, BRASIL
}

\author{
Danilo Fernandes da SILVA FILHO, Hiroshi NODA², Charles Roland CLEMENT2, \\ Francisco Manoares MACHADO'2.
}

RESUMO - O quebra-pedra (Phyllanthus stipulatus (Raf.) Webster, Euphorbiaceae) é um importante remédio popular usado para reduzir o ácido úrico no sangue e facilitar a eliminação de cálculos renais. Para avaliar a produção de biomassa dessa espécie utilizou-se sementes de populações naturais num experimento realizado em Manaus, AM. Adotou-se um delineamento experimental de parcelas subdivididas, onde as parcelas foram: (a) o ambiente natural e (b) o ambiente com tela plástica sombrite com $50 \%$ de luminosidade, e as subparcelas constituidas por dosagens de $0,2,4,6,8$ e $10 \mathrm{~kg}$ de composto orgânico/ $\mathrm{m}^{2}$. Nào foram encontradas diferenças significativas entre os ambientes na biomassa total das plantas $(63,1$ $\mathrm{g}$ vs $62,3 \mathrm{~g}$ fresca e $26,6 \mathrm{~g}$ vs $25,6 \mathrm{~g}$ seca), embora tivessem sido encontradas para a altura $(70,0 \mathrm{~cm}$ a pleno sol vs $96,2 \mathrm{~cm}$ sombra) e, conseqüentemente, para a biomassa do caule $(5,1 \mathrm{~g}$ vs $5,7 \mathrm{~g}$ seca, respectivemente). $\mathrm{O}$ quebra-pedra responde bem a adubação orgânica, tanto na biomassa total, como em todas as partes da planta. A melhor resposta, em termos de rendimento, se deu sob o efeito de $10 \mathrm{~kg}$ de $\mathrm{CO} / \mathrm{m}^{2}$ incorporado ao solo $\left(1,26 \mathrm{~kg} / \mathrm{m}^{2}\right.$ de biomassa fresca e $0,55 \mathrm{~kg} / \mathrm{m}^{2}$ de biomassa seca). Em comparação com a testemunha, este tratamento produziu $43 \%$ mais biomassa seca total. No entanto, a razào beneficio/ custo sugere que $4 \mathrm{~kg}$ de $\mathrm{CO} / \mathrm{m}^{2}$ é a quantidade máxima que é economicamente viável no solo usado. A biomassa total é composta de $17,2 \%$ de raizes, $22,3 \%$ de caules, $23,1 \%$ de galhos e $37,4 \%$ de folhas. $O$ crescimento das plantas exige muito potássio e pouco fósforo, magnésio e micronutrientes.

Palavras-chave: Quebra-pedra, Phyllanthus stipulatus, Euphorbiaceae, planta medicinal, agricultura alternativa, composto orgânico.

Effect of Organic Manure on Biomass Production of Quebra-Pedra (Phyllanthus stipulatus, Euphorbiaceae) in Manaus, Amazonas, Brazil.

ABSTRACT - Quebra-pedra (Phyllanthus stipulatus (Raf.) Webster, Euphorbiaceae) is a popular remedy used to reduce uric acid in blood and to facilite the elimination of kidney stones. The research was carried out at the Vegetable Crops Experiment Station of the National Institute for Amazonian Research, in Manaus, Amazonas, Brazil, using seeds of a local wild population. A split-plot design was used, where the main plots were the natural environment and $50 \%$ shade, the subplots were constituted by doses of $0,2,4,6,8$ and $10 \mathrm{~kg}$ of organic compost $(\mathrm{OC}) / \mathrm{m}^{2}$ and contained 20 plants in $1 \mathrm{~m}^{2}$. The following characters of the 6 useful plants per subplot were evaluated: stalk diameter at soil level, plant height, root, stalk, branch and leaf biomass (fresh and dry). No significant differences among environments were found for total plant biomass ( $63.1 \mathrm{~g}$ vs $62.3 \mathrm{~g}$ fresh wt, $26.6 \mathrm{~g}$ vs $25.6 \mathrm{~g}$ dry wt, respectively), but were found for height ( 70.0 $\mathrm{cm}$ in sun light vs $96.2 \mathrm{~cm}$ in shade) and, consequently, for stalk biomass (5.1 and $5.7 \mathrm{~g}$ dry wt, respectively). Quebra-pedra responds to organic manure, both in total biomass and in all of its components, with an increment of $43 \%$ in total biomass between the minimum and maximum treatment, but the benefit/cost ratio suggests that $4 \mathrm{~kg}$ of $\mathrm{OC} / \mathrm{m}^{2}$ is the maximum amount that is economically viable in the soil used. Total biomass is composed of $17.2 \%$ root, $22.3 \%$ stalk, $23.1 \%$ branches and $37.4 \%$ leaves. Plant growth demands considerable potassium but little phosphorous, magnesium or micronutrients.

Key-words: Quebra-pedra, Phyllanthus stipulatus, Euphorbiaceae, medicinal plant, alternative agriculture, organic compost.

P. P. I. N N 3360 "Coleta, Presevação, Caracterização e Uso de Germoplasma Tropical".

2 Coordenação de Pesquisas em Ciências Agronômicas, Instituto Nacional de Pesquisas da Amazônia, Cx. Postal 478, 69.011-970 Manaus, Amazonas, Brasil. 


\section{INTRODUÇÃO}

As plantas medicinais são usadas pelas populações indígenas e caboclas e por uma grande parte das pessoas nas áreas rurais e urbanas de cidades mais prósperas de qualquer país. Recentemente houve um aumento repentino no uso de plantas medicinais (FARNSWORTH, 1985). Por isso, o assunto tem recebido ampla cobertura da imprensa e de publicações leigas, muitas vezes não críticas ou comprovadas e algumas vezes até perigosas. Entretanto, para se garantir a segurança do uso de plantas medicinais ou remédios derivados delas, são necessários não apenas medidas de controle sobre a sua qualidade, mas também um esforço substancial para informar o público sobre todos os aspectos importantes de seu uso, desde o seu sistema de cultivo até o processamento e possiveis efeitos danosos que elas podem causar.

O quebra-pedra (Phyllanthus stipulatus (Raf.) Webster, Euphorbiaceae) é uma planta anual, herbácea, de pequeno porte e de folhas miúdas (JOLY, 1991). Esta espécie cresce espontaneamente em qualquer época do ano nos mais variados ambientes do Estado do Amazonas. Assim como acontece com outras espécies de Phyllanthus, as raizes ou a planta inteira são usadas na medicina popular em forma de chá ou de cápsulas industrializadas. O principio ativo da planta, é considerado antiespasmódico, relaxante muscular e muito eficiente na eliminacão do ácido úrico e cálculos renais pela urina (STASI et al., 1983; CORREAA, 1984; MATOS, 1994).
Nos países em desenvolvimento, somente uma pequena porção da matéria prima oriunda de plantas medicinais é produzida através de cultivo. Basicamente, a obtenção desse material é feita por meio do extrativismo. Quando cultivado, a grande maioria das pessoas utilizam sistemas agrícolas tradicionais (JUNIOR et al., 1994), ou seja, sem insumos agricolas comerciais (adubo químico, pesticidas, etc.). Por isso, a adoção de um modelo de agricultura alternativa, com o uso de composto orgânico, é uma boa opção para os pequenos agricultores da Amazônia; além da produção desse fertilizante estar ao alcance de todos, é considerada uma prática fitotécnica ecologicamente equilibrada e altamente sustentável (PASCHOAL, 1994). Dessa forma, será possível que os agricultores consigam produzir plantas saudáveis, livres de agrotóxicos e capazes de preservar todos os seus constituintes químicos.

No Estado do Amazonas já existem alguns resultados de pesquisas com a utilização de várias fontes alternativas de matéria orgânica. As experiências foram feitas com espécies nativas ou introduzidas na região e que podem ser usadas como alimento e/ou medicamento. SILVA FILHO et al. (1988) aplicaram $12 \mathrm{~kg} / \mathrm{m}^{2}$ de um composto orgânico e conseguiram um rendimento de $3,2 \mathrm{~kg}$ de folhas comestiveis de cariru (Amaranthus flavus) no primeiro corte, contra $0,56 \mathrm{~kg}$ sem o uso de fertilizante. NODA et al. (1978) empregaram matupá (uma mistura de macrófitas aquáticas tirada de ilhas flutuantes dos rios de água branca) 
como fertilizante orgânico na producão de feijão de asa (Psophocarpus tetragonolobus) e obtiveram um rendimento de 7,85 toneladas de sementes secas por hectare, enquanto a testemunha produziu somente 4,70 toneladas por hectare. PAHLEN (1977) usou composto orgânico para aumentar o rendimento de frutos de cubiu em mais de $400 \%$. SILVA FILHO et al. (1992) aplicaram $20 \mathrm{~kg}$ de um composto orgânico/ $/ \mathrm{m}^{2}$ em solo Podzólico e conseguiram um incremento de $542 \%$ de matéria seca de coentro (Coriandrum sativum) em relação à testemunha.

Este trabalho tem o objetivo de avaliar o comportamento de germoplasma local de quebra-pedra sob o efeito de dois ambientes e diferentes dosagens de um composto orgânico, com a intenção de orientar produtores locais.

\section{MATERIAL E MÉTODOS}

O ensaio foi conduzido na Estação Experimental de Hortaliças (EEH) do Instituto Nacional de Pesquisas da Amazônia (INPA), localizada no $\mathrm{km} 14$ da Rodovia AM-010, em Manaus, Amazonas, Brasil. O solo dessa área é um Podzólico Vermelho-Amarelo, álico, textura arenosa, de baixa fertilidade. O clima local é caracterizado com "Afi" no esquema de Köppen, registrando $2.450 \mathrm{~mm}$ de chuva, com uma estação seca no período de julho a setembro (RIBEIRO, 1976).

O preparo do solo foi feito manualmente, com o uso de enxada e ancinho para revolver e nivelar o solo. Foram construídos dois canteiros com $6 \mathrm{~m}$ de comprimento, $1 \mathrm{~m}$ de largura e $0,20 \mathrm{~m}$ de altura, em dois ambientes diferentes: I) a pleno sol e II) em casa de vegetação coberta com tela plástica sombrite com $50 \%$ de luminosidade. Amostras de solo das duas áreas foram coletadas e analisadas no Laboratório de Fertilidade de Solos do INPA, seguindo a metodologia da EMBRAPA (1979).

Utilizou-se sementes de quebrapedra que ocorrem naturalmente na EEH. A semeadura foi realizada no dia 25 de junho de 1995 e o transplante das mudas para o local definitivo no dia 24 de julho do mesmo ano.

Como fertilizante utilizou-se doses de $0,2,4,6,8$ e $10 \mathrm{~kg} / \mathrm{m}^{2}$ de um composto orgânico $(\mathrm{CO})$, cujo sistema de compostagem foi o de empilhamento de camadas alternadas de materiais vegetais e esterco bovino (como inoculante), sendo deixado em processo de fermentação por 90 dias. Amostras desse material foram analisadas no mesmo laboratório (Tab.1).

Adotou-se um delineamento experimental de parcelas subdivididas, com três repetições, com as parcelas constituídas pelos ambientes I e II e as sub-parcelas pelas doses crescentes de CO Cada subparcela continha 20 plantas num espaçamento de $0,20 \mathrm{~m} \mathrm{x}$ $0,20 \mathrm{~m}$, ocupando $1 \mathrm{~m}^{2}$.

A coleta foi realizada no dia 26 de janeiro de 1996, inicio da fase reprodutiva das plantas. Nas 6 plantas úteis de cada subparcela avaliou-se os seguintes caracteres: diâmetro do caule, altura, pesos fresco e seco das raizes, caules, galhos e folhas. As mesmas plantas serviram de amostras para análise dos seus constituintes minerais. A regressão da biomassa total sobre as doses de $\mathrm{CO}$ foi obtida a 
partir das médias desses caracteres no ambiente a pleno sol.

\section{RESULTADOS E DISCUSSÃO}

Os teores de fósforo disponível no solo (Tab. 1) foram elevados para um solo do tipo Podzólico VermelhoAmarelo da região, onde $\mathrm{P}$ varia de 0 a $3 \mathrm{mg} \cdot \mathrm{dm}^{-3}$ (SANCHES et al., 1982). A explicação é que nas duas areas experimentais, os solos foram adubados para o cultivo de outras hortaliças antes do experimento com quebra-pedra, permanecendo o efeito residual no solo, Os níveis de matéria orgânica do solo e de macro e microelementos encontrados no $\mathrm{CO}$ são considerados de médio a alto quando comparados com as referências de MALAVOLTA (1992).

Como era de se esperar, o teor de alumínio do CO foi "zero", valor geralmente observado em análises de compostos orgânicos (KIEHL, 1985).

A alta concentração dos elementos $\mathrm{K}, \mathrm{Ca}, \mathrm{Mg}$ no CO (Tab.1) está relacionada com a qualidade da matéria-prima utilizada na preparação do fertilizante. De acordo com KIEHL (1985), a riqueza de um composto orgânico em nutrientes está estreitamente relacionada com a natureza dos vegetais e dos inoculantes que lhe deram origem. Já que o CO preparado na EEH do INPA utiliza uma grande variedade de restos de frutos, hortaliças, vagens de algumas leguminosas, folhas diversas, etc, tem tudo para se constituir num adubo de boa composição mineral.

Entre os caracteres avaliados, só o diâmetro do caule não foi afetado pelas doses de $\mathrm{CO}$ ou pelos ambientes (Tab.
2). A altura da planta apresentou um efeito significativo dos ambientes, com maior crescimento na sombra, sugerindo estiolamento provocado por uma insuficiência de luz. Isso também sugere que o desenvolvimento em altura das plantas, sem o acúmulo de biomassa total, pode ser explicado pela plasticidade alométrica (RODRIGUEZ FUENTES et al., 1987). Os componentes da biomassa fresca e seca sujeitos a estiolamento (caule e galhos) apresentaram diferenças significativas entre os ambientes, bem como entre os tratamentos. Entretanto as raízes e folhas não apresentaram diferenças entre os ambientes.

Observou-se que à proporção que aumentou a quantidade de $\mathrm{CO}$ adicionada ao solo, aumentou linearmente a produção de biomassa nas diferentes partes da planta nos dois ambientes estudados (Tab. 2, Fig.1). O quebra-pedra responde bem a adubação orgânica, tanto na biomassa total como em todos os componentes da planta, com um incremento de $43 \%$ na biomassa total entre os tratamentos mínimo e máximo (19,1 e 27,3 g seca, respectivamente). Com a aplicação de $10 \mathrm{~kg}$ de $\mathrm{CO} / \mathrm{m}^{2}$, o rendimento em biomassas fresca e seca alcançou $1,26 \mathrm{~kg}$ e $0,55 \mathrm{~kg} / \mathrm{m}^{2}$, respectivamente. Em comparação com a testemunha, este tratamento foi superior em produção de raizes, caules, galhos e folhas na ordem de $16,8 \%, 27,1 \%, 61,5 \%$ e $61,3 \%$, respectivamente.

A aplicação de 1 a $5 \mathrm{~kg}$ de matéria orgânica $/ \mathrm{m}^{2}$ é recomendada para a produção de biomassa de várias espécies medicinais (JUNIOR et al., 1994). Entretanto, é importante verificar se o uso desse fertilizante é viável economicamente 
Tabela 1. Composição química do solo e do composto orgânico usados como substrato para avaliar a produção de quebra-pedra em Manaus, Amazonas, Brasil, em 1996.

\begin{tabular}{|c|c|c|c|c|c|c|c|c|c|}
\hline \multirow[b]{2}{*}{ Amostra } & \multirow{2}{*}{$\begin{array}{l}\mathrm{pH} \\
\text { água }\end{array}$} & $\mathrm{C}$ & $\mathrm{N}$ & \multirow{2}{*}{$\frac{\mathrm{P}}{\mathrm{mg} / \mathrm{kgg}}$} & $\mathrm{K}$ & $\mathrm{Ca}$ & $\mathrm{Mg}$ & $\mathrm{Na}$ & $\mathrm{Al}$ \\
\hline & & \multicolumn{2}{|c|}{$\mathrm{g} / \mathrm{kg}$} & & \multicolumn{5}{|c|}{$\mathrm{cmol} \mathrm{kg}$} \\
\hline Amb. I & 5,40 & 2,07 & 0,07 & 71 & 0,10 & 0,80 & 0,49 & 0,15 & 0,05 \\
\hline Amb. II & 5,47 & 2,22 & 0,07 & 111 & 0,07 & 2,14 & 0,51 & 0,18 & 0,02 \\
\hline Composto & 5,41 & 15,48 & 0,61 & 118 & 1.38 & 5,45 & 2,27 & 0,33 & 0,00 \\
\hline
\end{tabular}

Tabela 2. Médias do diâmetro, da altura, das biomassas fresca e seca total e das diferentes partes da planta ( $\mathrm{g} /$ planta) de quebra-pedra avaliadas sob o efeito de composto orgânico a pleno sol e sob 50\% de sombra em Manaus, Amazonas, Brasil, em 1996.

\begin{tabular}{|c|c|c|c|c|c|c|c|c|c|c|c|c|}
\hline \multirow{3}{*}{$\begin{array}{l}\text { Trat } \\
\mathrm{kg} / \mathrm{m}^{2}\end{array}$} & \multirow{2}{*}{\multicolumn{2}{|c|}{$\begin{array}{c}\text { Diämetro } \\
(\mathrm{mm})\end{array}$}} & \multirow{2}{*}{\multicolumn{2}{|c|}{$\begin{array}{l}\text { Altura } \\
(\mathrm{cm})\end{array}$}} & \multicolumn{4}{|c|}{ Biomassa Total } & \multirow{2}{*}{\multicolumn{4}{|c|}{$\begin{array}{c}\text { Biomassa Seca Total } \\
\text { (a pleno sol) }\end{array}$}} \\
\hline & & & & & \multicolumn{2}{|c|}{ Fresca } & \multicolumn{2}{|c|}{ Seca } & & & & \\
\hline & I & II & I & II & I & II & 1 & II & Raiz & Caule & Galho & Folha \\
\hline 0 & 3,9 & 3,1 & $54,2 \mathrm{aA}$ & $90,8 \mathrm{~B}$ & $41,2 \mathrm{a}$ & 39,8 & $19,1 \mathrm{a}$ & 20,4 & $4,2 \mathrm{a}$ & $4,8 \mathrm{a}$ & $3,9 a$ & $6,2 a$ \\
\hline 2 & 4,9 & 4,8 & $54,5 \mathrm{aA}$ & $91,9 \mathrm{~B}$ & $44,2 \mathrm{ab}$ & 43,5 & $21,1 \mathrm{ab}$ & 20,6 & $4,3 \mathrm{a}$ & $5,1 \mathrm{a}$ & $4,4 a b$ & $7,3 \mathrm{ab}$ \\
\hline 4 & 4,3 & 3,9 & $61,6 \mathrm{abA}$ & $92,0 \mathrm{~B}$ & $46,6 \mathrm{ab}$ & 46,5 & $22,0 \mathrm{ab}$ & 21,1 & $4,5 b$ & $5,3 \mathrm{ab}$ & $4,5 \mathrm{ab}$ & $7,6 a b$ \\
\hline 6 & 4,8 & 4,7 & $61,7 \mathrm{abA}$ & $94,3 \mathrm{~B}$ & $49,0 \mathrm{ab}$ & 47,5 & $24,2 \mathrm{bc}$ & 22,9 & $4,6 b$ & $5,3 \mathrm{ab}$ & $6,6 \mathrm{~b}$ & $7,7 \mathrm{ab}$ \\
\hline 8 & 5,0 & 5,0 & $65,2 \mathrm{bA}$ & $95,7 \mathrm{~B}$ & $54,0 \mathrm{ab}$ & 52,6 & $24,9 b c$ & 23,5 & $4,7 b$ & $6,0 \mathrm{~b}$ & $5,0 \mathrm{~b}$ & $9,1 b$ \\
\hline 10 & 5,6 & 5,5 & $70,0 \mathrm{bA}$ & $96,2 \mathrm{~B}$ & $63,11 \mathrm{~b}$ & 62,3 & $27,3 \mathrm{c}$ & 25,7 & $4,9 b$ & $6,1 a b$ & $6,3 c$ & $10,0 \mathrm{~b}$ \\
\hline
\end{tabular}

* As médias seguintes pela mesma letra minúscula na coluna e maiúscula na linha nảo diferem significativamente ao nivel de $5 \%$ pelo teste de Tukey

1-Ambiente a pleno sol; II-Ambiente sombreado com $50 \%$ de luminosidade.

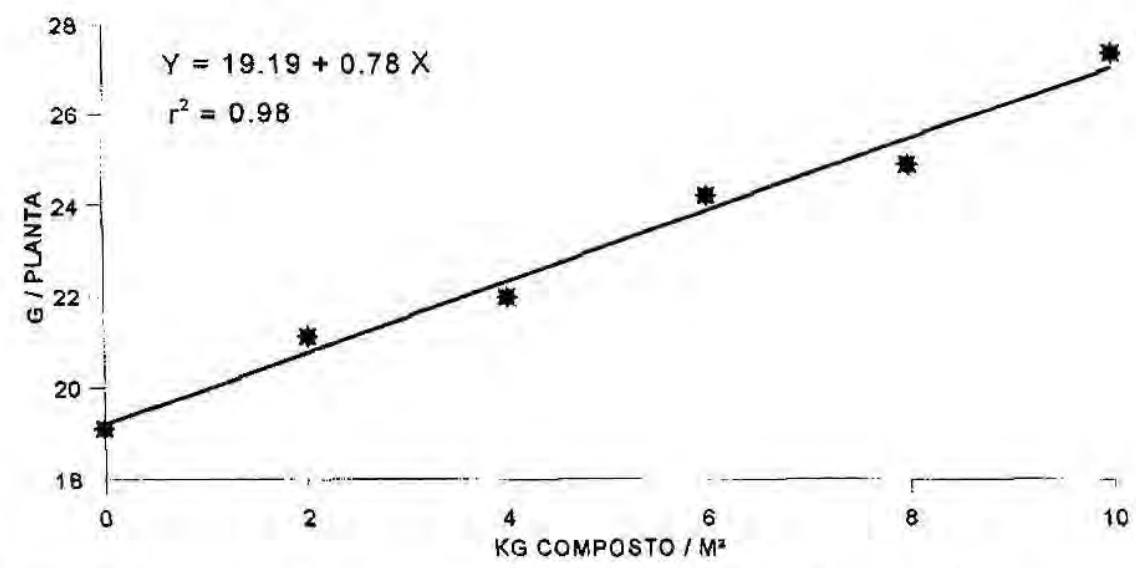

Figura 1. Relação entre a quantidade de composto orgânico usada por $\mathrm{m}^{2} \mathrm{e}$ a biomassa seca total ( $\mathrm{g} / \mathrm{planta}$ ) produzida. A resposta ao composto é linear no intervalo de quantidades usadas, sugerindo que a assintote não esteja incluida no intervalo 
no cultivo de quebra-pedra. Considerando que um $1 \mathrm{~kg}$ de $\mathrm{CO}$ fabricado na Estação Experimental do INPA custa R $\$ 0,12$ e que o valor de $1 \mathrm{~kg}$ de quebra-pedra no atacado (processado, pronto para o uso industrial) é de $\mathrm{R} \$ 4,50$, o emprego de $4 \mathrm{~kg}$ de $\mathrm{CO}$ / $\mathrm{m}^{2}$ se justifica economicamente porque a razão beneficio/custo sugere um retorno de mais de $20 \%$ (Tab. 3). No entanto, estes cálculos precisam ser usados com cautela, tendo em vista que o solo onde foi realizado o experimento contém resíduos nutricionais importantes.

Tanto na medicina popular quanto na indústria de produtos homeopáticos usa-se a planta inteira do quebra-pedra no preparo de medicamentos. No entanto, é possivel que uma das partes da planta possa ser mais utilizada como matéria-prima. Proporcio-nalmente, uma planta de quebra-pedra apresenta $17,2 \%$ de raiz, $22,3 \%$ de caule, $23,1 \%$ de galhos e $37,4 \%$ de folhas. Considerando que a análise econômica foi feita em função da utilização da planta inteira, seria aconselhável recalcular a razão beneficio/custo no caso de apenas uma das diferentes partes da planta venha ser usada.

O quebra-pedra parece ser pouco exigente em fósforo, magnésio e micronutrientes, já que as quantidades
Tabela 3. A razão beneficio/custo de produçâo de quebra-pedra sob o efeito de composto orgânico (CO) a pleno sol em Manaus, Amazonas, Brasil, em 1996. $(\mathrm{R} \$ 1,02=$ US\$1 $)$

\begin{tabular}{ccccc}
\hline $\begin{array}{c}\text { Tratamento } \\
\left(\mathrm{kg} \mathrm{CO} / \mathrm{m}^{2}\right)\end{array}$ & $\begin{array}{c}\text { Produçào } \\
\left(\mathrm{kg} \mathrm{seco} / \mathrm{m}^{2}\right)\end{array}$ & $\begin{array}{c}\text { Beneficio } \\
\left(\mathrm{RS} / \mathrm{m}^{2}\right)\end{array}$ & $\begin{array}{c}\text { Custo } \\
\left(\mathrm{RS} / \mathrm{m}^{2}\right)\end{array}$ & Razào B/C \\
\hline 0 & 0,382 & 1,72 & 1,02 & 1,68 \\
2 & 0,422 & 1,90 & 1,28 & 1,48 \\
4 & 0,438 & 1,97 & 1,53 & 1,29 \\
6 & 0,444 & 2,00 & 1,77 & 1,13 \\
8 & 0,496 & 2,23 & 2,04 & 1,09 \\
10 & 0,546 & 2,46 & 2,46 & 1,07 \\
\hline
\end{tabular}

destes nutrientes mostraram pouca variação à medida que aumentou a quantidade de $\mathrm{CO}$ (Tab. 4). Ao mesmo tempo, parece ser moderadamente exigente em cálcio e muito exigente em potássio. A forte extração de potássio do solo poderia estar relacionada com as várias funções desse elemento na planta, entre as quais estão o aumento da resistência a seca, a pragas e doenças, conferindo-lhe rusticidade. Por outro lado, MALAVOLTA (1989) faz referência ao consumo, em algumas espécies, de elevados níveis de $\mathrm{K}$ sem que as plantas realmente necessitem. Isto é chamado de consumo de luxo. No entanto, para confirmar estas suposições, será necessário um experimento direcionado especificamente para estudar as exigências nutricionais do quebra-pedra.

Tabela 4. Níveis médios de nutrientes contidos em 20 plantas de quebra-pedra cultivadas sob o efeito de composto orgânico a pleno sol e sob 50\% de sombra em Manaus, Amazonas, Brasil, em 1996.

\begin{tabular}{|c|c|c|c|c|c|c|c|c|}
\hline \multirow{2}{*}{$\begin{array}{c}\text { Trat. } \\
\left(\mathrm{kg} / \mathrm{m}^{2}\right)\end{array}$} & $\mathrm{P}$ & $\mathrm{K}$ & $\mathrm{Ca}$ & $\mathrm{Mg}$ & $\mathrm{Fe}$ & $\mathrm{Zn}$ & $\mathrm{Cu}$ & $\mathrm{Mn}$ \\
\hline & \multicolumn{4}{|c|}{ (g/20 plantas) } & \multicolumn{4}{|c|}{ ( $\mathrm{mg} / 20$ plantas) } \\
\hline 0 & 1.25 & 4.16 & 1.79 & 1.40 & 375 & 41 & 5.7 & 27 \\
\hline 2 & 1.24 & 4.35 & 1.80 & 1.45 & 426 & 43 & 5.7 & 29 \\
\hline 4 & 1.28 & 6.42 & 1.92 & 1.76 & 459 & 45 & 5.0 & 34 \\
\hline 6 & 1.32 & 6.88 & 1.98 & 1.51 & 474 & 48 & 5.9 & 35 \\
\hline 8 & 1.33 & 7.29 & 2.00 & 1.51 & 672 & 50 & 5.9 & 39 \\
\hline 10 & 1.35 & 9.02 & 2.38 & 1.71 & 679 & 52 & 6.3 & 4 \\
\hline
\end{tabular}




\section{CONCLUSÕES}

$\mathrm{O}$ ambiente sombreado favorece $\mathrm{o}$ crescimento do quebra-pedra em altura mas sem o acúmulo adicional de biomassa.

$\mathrm{O}$ quebra-pedra responde à adubação orgânica. Quando cultivado a pleno sol, o maior incremento em biomassas fresca e seca $\left(1,26 \mathrm{~kg} / \mathrm{m}^{2}\right.$ e $0,55 \mathrm{~kg} / \mathrm{m}^{2}$, respectivamente) pode ser conseguido com a adição de $10 \mathrm{~kg} / \mathrm{m}^{2}$ de composto orgânico.

A maior parte da biomassa do quebra-pedra $(37,4 \%)$ se concentra nas folhas.

\section{AGRADECIMENTOS}

Os autores agradecem ao técnico José Edivaldo Chaves, CPCA-INPA, pela realização das análises de solo, da matéria orgânica e das plantas utilizadas no experimento, e aos dois "referees" da Acta Amazonica pelas criticas e sugestões que enriqueceram a qualidade do trabalho.

\section{Bibliografia Citada}

CORRÊA, M.P. 1984, Dicionário das plantas úteis do Brasil e das exóticas cultivadas. Rio de Janeiro: Imprensa Nacional, v.6.

EMBRAPA. 1979. Manual de métodos de análise de solo. Rio de Janeiro: Serviço Nacional de Levantamento de Solos. 98p.

FARNSWORTH, N.R. 1985. Medicinal plants in therapy. Bulletin of the World Health Organization, 63: 965-981.

JOLY, A.B. 1991. Introdução à taxonomia vegetal, $10^{\underline{a}}$ ed. São Paulo: Editora da Universidade de São Paulo. $177 \mathrm{p}$.

JUNIOR, C.C.; MING, L.C.; SCHEFFER, M.C. 1994. Cultivo de plantas medicinais, condimentares e aromáticas, $2^{2}$ ed. Jaboticabal, SP: Fundação de Estudos e Pesquisas em Agronomia e Medicina Veterinária e Zootecnia. $162 \mathrm{p}$.
KIEHL, E.J. 1985. Fertilizantes orgânicos. Piracicaba: Editora "Ceres". 492p.

MALAVOLTA, E;; VITTI, G.C.; OLIVEIRA, S.A. 1989. Avaliação do estado nutricional das plantas: principios $e$ aplicações. Piracicaba, SP: Ass. Bras. para Pesq. da Potassa e do Fosfato. 201p.

MALAVOLTA, E. 1992. ABC da análise de solos e folhas. São Paulo: Editora Agronômica "Ceres" Ltda. 124p.

MALAVOLTA, E.; VITTI, GODOFREDO; OLIVEIRA, S.A. 1989. Avaliação do estado nutricional das plantas: principios e aplicações. Piracicaba: Ass. Bras, para Pesq.da Potassa e do Fosfato. 201p.

MATOS, F.J.A. 1994. Farmácias vivas, sistema de utilização de plantas medicinais projetado para pequenas comunidades, $2^{\mathrm{a}}$ ed. Fortaleza: Editora da Universidade Federal do Ceará. 180p.

NODA, H.; JUNK, W.J.; PAHLEN, A. von der. 1978. Emprego de macrófitas aquáticas (matupá) como fonte de matéria orgânica na cultura do feijão de asa (Psophocarpus tetragonolobus) em Manaus. Acta Amazônica, 8(1): 107-109.

PAHLEN, A. von der. 1977. Cubiu (Solanum topiro Humb. \& Bonpl.), uma fruteira da Amazônia. Acta Amazônica, 7: 301-307.

PASCHOAL, A.D. 1994. Produção orgânica de alimentos: agricultura sustentável para o século XX e XXI. Piracicaba, SP: Escola Superior de Agricultura "Luiz de Queiroz," USP. 191p.

RIBEIRO, M.N.G. 1976. Aspectos climatológicos de Manaus. Acta Amazônica, 6(2): 229-233.

RODRIGUEZ FUENTES, C.; PÉREZ, P.J.; FUCHA, A. 1987. Genética y mejoramiento de las plantas. Ciudad de La Habana: Editorial Pueblo y Educación. 224p.

SILVA FILHO, D.F.; NODA, H; CLEMENT, C.R.; NODA, S.N. 1988. Efeito da matéria orgânica na produção de biomassa de cariru. Horticultura Brasileira, 6(1): 80 .

SILVA FILHO, D.F; NODA, H.; WANDERLEY, L.J.G. 1992. O comportamento do coentro (cv. Verdão) em solo podzólico vermelhoamarelo na região de Manaus. Horticultura Brasileira, 8(1): 136. 
SANCHEZ, P.A.; BANDY, D.E.; VILLACHICA, J.H.; NICHOLAIDES, J.J. 1982. Amazon basin soils: Management for continuous crop production. Science, 216: 821-827.
STASI, L.C.; SANTOS, E.M.G.; SANTOS, C.M. dos; HIRAMA, C.A. 1989. Plantas medicinais na Amazônia. SÀo Paulo; UNESP. 141p. 